\title{
(- OPEN ACCESS \\ Addressing complexity in population health intervention research: the context/intervention interface
}

\author{
Laetitia Minary, ${ }_{1,2,3}$ François Alla, ${ }^{1,3,4}$ Linda Cambon, ${ }^{1,5,6}$ Joelle Kivits, ${ }^{1,4}$ \\ Louise Potvin $2,7,8$
}

${ }^{1}$ EA4360 Apemac, Université de Lorraine, Université Paris Descartes, Vandoeuvre les Nancy, Nancy, France

${ }^{2}$ Département de Médecine Sociale et Préventive, Ecole de Santé Publique, Universite de Montreal Faculte de medecine, Montréal, Canada ${ }^{3}$ Centre Hospitalier Universitaire de Nancy, INSERM CIC-EC Nancy, France

${ }^{4}$ Université de Lorraine, Ecole de Santé Publique, Nancy, France ${ }^{5}$ EHESP, Chaire Recherche en Prévention des Cancer, Rennes, France

${ }^{6}$ CNRS, UMR CRAPE Centre de Recherche sur l'Action Politique en Europe-6051, Rennes, France Institut de Recherche en Santé Publique de I'Université de Montréal, Université de Montréal, Montreal, Quebec, Canada

${ }^{8}$ Centre de Recherche LéaRoback sur les Inégalités Sociales de Santé de Montréal, Montréal, Quebec, Canada

\section{Correspondence to}

Dr Laetitia Minary, EA 4360 APEMAC, Faculté de Médecine Université de Lorraine Université Paris Descartes, Vandoeuvre-Les-Nancy 54505, France; laetitia.minary@univlorraine.fr

Received 30 August 2017 Revised 17 November 2017 Accepted 21 November 2017 Published Online First 10 January 2018

Check for updates

To cite: Minary L, Alla F, Cambon L, et al. J Epidemiol Community Health 2018:72:319-323.

\section{ABSTRACT \\ Background Public health interventions are} increasingly being recognised as complex and context dependent. Related to this is the need for a systemic and dynamic conception of interventions that raises the question of delineating the scope and contours of interventions in complex systems. This means identifying which elements belong to the intervention (and therefore participate in its effects and can be transferred), which ones belong to the context and interact with the former to influence results (and therefore must be taken into account when transferring the intervention) and which contextual elements are irrelevant to the intervention.

Discussion This paper, from which derives criteria based on a network framework, operationalises how the context and intervention systems interact and identify what needs to be replicated as interventions are implemented in different contexts. Representing interventions as networks (composed of human and non-human entities), we introduce the idea that the density of interconnections among the various entities provides a criterion for distinguishing core intervention from intervention context without disconnecting the two systems. This differentiates endogenous and exogenous intervention contexts and the mediators that connect them, which form the fuzzy and constantly changing intervention/context interface.

Conclusion We propose that a network framework representing intervention/context systems constitutes a promising approach for deriving empirical criteria to delineate the scope and contour of what is replicable in an intervention. This approach should allow better identification and description of the entities that have to be transferred to ensure the potential effectiveness of an intervention in a specific context.

Public health interventions in the form of policies and programmes are designed to modify the distribution of health determinants in a population. ${ }^{1}$ Shiell et $a l^{2}$ proposed a systemic approach to dealing with the complexity of such interventions. According to them, an intervention can be represented as a series of inter-related events occurring within a broader system that they are in constant interaction with. Intervention effects are thus modulated-attenuated or amplified-by the characteristics and dynamic evolution of the system (context) in which it is implemented. ${ }^{3}$ The observed effects are therefore attributable to the specific characteristics of the intervention and are influenced by the implementation context. In addition, interventions are adaptive, so they evolve as a result of transformations in context. ${ }^{4}$

This intervention/context dependency raises the issue of distinguishing between what belongs solely to the intervention-and can be transferred to other contexts-and what belongs to the implementation context. This distinction is important for programme evaluation purposes. First, since adaptive interventions are dynamic and changing, their evaluation must take these changes into account in the longitudinal perspective of implementation. ${ }^{4}$ Second, intervention effects are the product of 'intervention by context' interactions, ${ }^{56}$ and evaluation must therefore open up the intervention 'black box' to understand which elements of the intervention interacted (and how they interacted) with which elements of the context in order to produce observed effects. Based on Hawe et al's ${ }^{3}$ proposition that interventions are related events in a system and on the Bilodeau and Potvin sociotechnical networks representation of the intervention, this paper further operationalises a network-based representation of population health interventions and proposes criteria for distinguishing what needs to be replicated as interventions are transferred.

\section{FUZZY BOUNDARY OF INTERVENTION AND CONTEXT}

There is a consensus that the content of public health interventions constitutes the aspects that must be preserved when interventions are transposed to another context. However, the conceptual and methodological ambiguities concerning constitutive intervention elements persist. The notion of key function is used to refer to a set of functions and processes designed to facilitate organisational/ structural changes. ${ }^{7}$ This concept is fairly close to the notion of an active ingredient in that it mainly refers to the theory of change process. ${ }^{8}$ The notion of a core element adds the implementation features of the intervention to this theoretical dimension. Finally, the broader notion of the component proposed by the UK Medical Research Council ${ }^{10}$ integrates all of these definitions. It designates various human and non-human entities that variously influence the intervention outcomes, which can be aggregated, and that produce emerging properties that cannot be reduced to their individual parts. ${ }^{11}$ Human entities are the intervention staff, participants, decision makers and other 
relevant human beings, whereas non-human entities are the financial resources, scientific knowledge and material, symbolic procedural artefacts constitutive of complex interventions.

Although the importance of context has been extensively discussed in the public health literature, its description in a robust and dynamic way remains rare. ${ }^{12}$ Most importantly, the ways in which interacts with an intervention has rarely been operationalised. However, taking the context into account cannot simply be limited to a description of the intervention beneficiaries' characteristics, the intervention time periods or the intervention location (eg, school, socioeconomic status of the inhabitants of the residential area or local urban facilities). ${ }^{12}{ }^{13}$ Poland et $a l^{14}$ defined context as the spatial and temporal conjunction of social events, individuals and social interactions, which generate causal mechanisms that interact with the intervention and can modify the intervention effects.

A recent review of the literature on context ${ }^{15}$ revealed that context is characterised as 'complex and dynamic, constantly changing, and rarely straightforward'. Context was perceived as a 'physical element or a non-physical construct existing within complex multilayered systems (...) and interacting not only with each other, but also with a broader environment, usually in non-linear ways'. The authors further emphasised that the interface or boundary between these elements is dynamic and fuzzy, reflecting the 'subjectivity of context, subjectivity not only related to individuals or entities, but also to the objects considered, in this case the intervention'.

Context can therefore be defined as:

A set of characteristics and circumstances that consist of active and unique factors that surround the implementation effort. As such, some attributes of context can be modified to make them an active component of the intervention to enhance implementation and effectiveness. It is therefore not a backdrop for implementation but interacts, influences, modifies and facilitates or constrains the intervention and the implementation effort. (...) Context is much more versatile, embracing not only the setting but also roles, interactions and relationships. ${ }^{15}$

Reiterating the major role of interactions and relationships to defining the context, Rod et al $^{16}$ specified how effective public health interventions reconfigure social relationships. According to them, an effective intervention changes its context, which in turn transforms the intervention. Accordingly, the authors proposed the 'spirit of intervention' concept to define the dimensions of an intervention that make it socially effective.

\section{THE KEY QUESTION: HOW TO DISTINGUISH THE INTERVENTION FROM ITS CONTEXT?}

Considering the adaptive and interactive dimensions of interventions in context, the literature invites us to understand the whole system instead of decomposing it into its individual components. ${ }^{3717}$ Some authors use the network metaphor to define an intervention as a system. Hawe $e t$ al associated such a system to an evolving network of person-time-place interactions that changes relationships, displaces existing activities and redistributes and transforms resources. ${ }^{3}$ They consider that an intervention might be best thought of as a time limited series of events, new activity settings and technologies that have the potential to transform the system because of their interaction with the context and the capability created from this interaction. Such approach implies 'to studying and understanding the context and specifically, to draw attention to the networks of social relationships that make up the system, the variety of roles that exist or can be created within those networks, the status conferred on those roles, the symbolism, and the meaning that different actors draw from the intervention event'. ${ }^{3}$ To illustrate their theoretical approach, Hawe et al operationalised how an intervention can transform the structure of the network of people in a community agency through the creation of new events and activities using social network analysis. However, their representation focus on social relationship and did not allow to distinguish intervention and context.

In their adaptation of the actor-network theory, ${ }^{17}$ Bilodeau and Potvin explained why interventions can be conceived as sociotechnical networks in which the differentiation between human and non-human, social and technical and individual and collective is irrelevant. Such an approach implies to focus on the connections between the various entities that compose the intervention and its context, their mutual influence and the evolution of the network they form. In line with Hawe et $a l^{3}$ and with Bilodeau and Potvin, ${ }^{17}$ we suggest that representing an intervention as a network composed of human and non-human entities provides an operational criterion to distinguish entities belonging to the intervention, to those belonging to the context as well as the interface between the two (see figure 1). Such model requires prior characterisation of the identity and roles of each entities and of their interconnections. This leads us to thus distinguish between an endogenous and an exogenous intervention context and the mediators that form the fuzzy and constantly changing interface between the two.

- The endogenous context is constituted of entities that are inter-related through dense and stable connections that form the core of the network.

- The exogenous context is composed of entities that exhibit lower density connections and that are not directly connected with the endogenous context. These exogenous contextual entities can potentially become connected to the endogenous context through their connections with mediators.

- Entities at the periphery of the endogenous contextual intervention network are the mediators through which the intervention is implemented in the context. They are ultimately connected with the entities in the exogenous context. Conversely, they also act as conduits through which the exogenous context interacts with the intervention's endogenous context. They compose a constantly changing and fuzzy border between an intervention and its context.

To illustrate this model, we propose the example of a nutrition education programme ${ }^{4}$ (Box 1 and figure 2) in which the nutritionist, knowledge and recipes are closely connected and participate to the core of the intervention's endogenous context. The community development workers are less connected to the rest of the core of the intervention's endogenous context and constitute entities of the exogenous context. The teachers form an interface between the endogenous and exogenous contexts (educational institution). They are mediators.

This model accounts for the context/intervention interconnections. Using the density of interconnections as a criterion, it provides an empirical approach to distinguishing between the entities that can be considered intervention specific and those that belong to the context without disconnecting the two. It could lead to an improvement in the transferability of public health interventions by identifying which core entities and interactions to foster when implementing an intervention that has previously been evaluated in another context. If we consider the example of the Petits cuistots-Parents en réseaux (PC-PR) programme (figure 2), the link between knowledge and the participating pupils seems to be important. Thus, in a transfer 


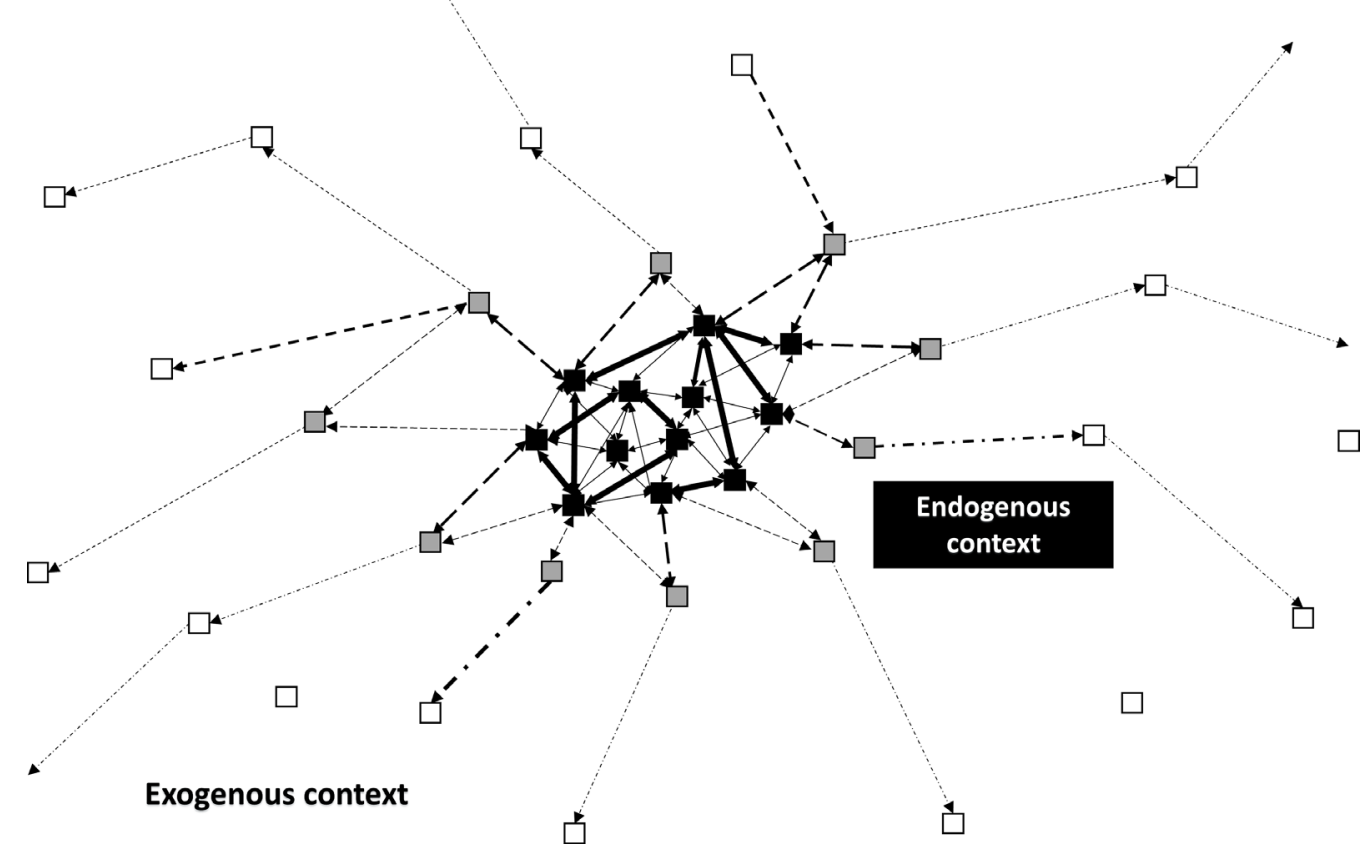

Figure 1 Graphic representation of the intervention/context system. The black squares characterise the entities specific to the endogenous context. The grey squares represent entities that connect the intervention's endogenous and exogenous contexts and allow interactions between the two. The white squares represent entities that the intervention's endogenous context is not directly connected with (exogenous context). The number of interconnections between entities characterises a network density, whereas the strength of the various connections is characterised by the thickness of the lines that connect entities.

process, attention should be paid to the way in which knowledge is appropriated by the students and particularly to the nutritionist's skills in providing this knowledge and their capacity to interact with the students. By contrast, if extracurricular activities were identified as not fundamental, they could potentially be removed if there are less financial and human resources. Finally,

\section{Box 1 Application of the model to the Petits cuistots-} parents en réseaux (PC-PR) programme

After identifying all the programme entities mentioned in the article, we elaborated a matrix based on all the interactions described. The network representation in figure 2 was created using the UCINET 6 software. It illustrates three key propositions stemming from our proposed model.

First, some entities are clearly more interconnected than others (at the periphery) and are characterised here as the programme's endogenous context.

Second, the sociotechnical network of the Petits cuistotsParents en réseaux (PC-PR) programme shows that the endogenous context connects human (nutritionist, students, teachers and parents) and non-human entities (recipes, knowledge, know-how, food, tasting samples and cooking utensils).

Third, the components at the periphery are the mediators and link the programme's endogenous context with its exogenous context. For example, the preparatory exercises link the PC-PR programme with the overall educational programme, which is part of the exogenous context. In addition, parents can also act as linking the PC-PR programme with other family members. such a model allows to better identify which transformations (integration or deletion of an entity or interaction) impacted the results of the intervention during its transfer (ie, its failure, strengthened success or unanticipated consequences).

This model constitutes a hypothesis for operationalisation of a systemic approach to intervention complexity in a perspective of intervention transfers. Empirical research is needed to validate and to define the dynamic properties of the interface between the interventions' endogenous and exogenous contexts. In view of the changing nature of systems, research is also needed to investigate the transformation of intervention systems over time or when the intervention is implemented in another context.

\section{CONCLUSION}

One of the challenges faced by public health research is informing actors and decision makers about the mechanisms underlying intervention effectiveness and the way in which an intervention can be adapted and transferred to another context. It is therefore essential to try to distinguish the entities that are specific to the intervention from those that are related to its context. We propose that a network framework representing intervention/context systems constitutes a promising approach to deriving empirical criteria that delineate the scope and contour of what is replicable. It is important now to conduct further research on theoretical and methodological developments and to validate this model through empirical studies. This approach should allow a better identification and description of the entities that have to be transferred to ensure the potential effectiveness of an intervention in a specific context. 


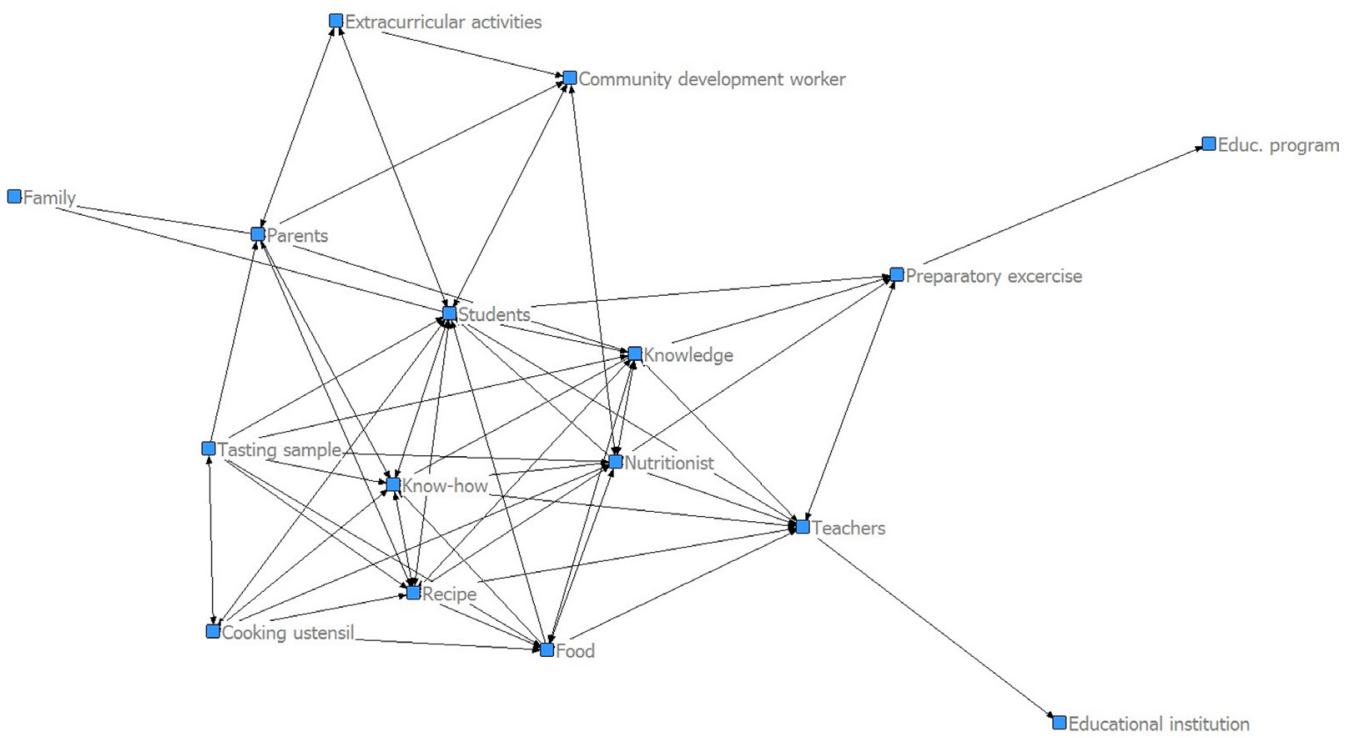

Figure 2 This example illustrates the model through the mapping of the components of the Petits cuistots-parents en réseaux (PC-PR) programme as presented in an implementation evaluation study of this programme. ${ }^{4}$

\section{What is already known on this subject}

- The observed effects of an intervention are attributable to the specific characteristics of the intervention and are influenced by the implementation context.

- This intervention/context dependency raises the issue of distinguishing between what belongs solely to the intervention-and can be transferred to other contexts - and what belongs to the implementation context.

- Representations of the network metaphor can be used to define an intervention as a system. However, its operationalisation still focus on social relationship and did not allow to distinguish intervention and context.

\section{What this study adds}

- This model allows us to distinguish an endogenous and exogenous intervention context and the mediators that form the fuzzy and constantly changing interface between these two contexts.

- It provides an empirical approach to operationalise the nesting of intervention within context without disconnecting the two.

- It helps identify which core entities and interactions to foster when transferring an intervention that has previously been evaluated in another context.

- It allows to identify which transformations (integration or deletion of an entity or interaction) impacted the results of the intervention during its transfer.

Acknowledgements We would like to thank Erica Di Ruggiero for comments that greatly improved the manuscript.

Contributors LM conceived the idea for the paper, wrote the first draft and led the writing of the paper. FA, LC and JK helped draft the manuscript. LP also conceived the idea for the paper and assisted with the writing of the manuscript. LM acts as guarantor. All authors have approved the final version.

Funding This work was supported by the French National Cancer Institute/French Public Health Research Institute /ARC Foundation for Cancer Research, grant
'Primary prevention 2014' and by the French League against Cancer, grant 'Research project in Epidemiology 2014'. LP holds the Canada Research Chair in Community Approaches and Health Inequalities (CIHR 950-228295).

Competing interests None declared.

Provenance and peer review Not commissioned; externally peer reviewed.

Open Access This is an Open Access article distributed in accordance with the Creative Commons Attribution Non Commercial (CC BY-NC 4.0) license, which permits others to distribute, remix, adapt, build upon this work non-commercially, and license their derivative works on different terms, provided the original work is properly cited and the use is non-commercial. See: http://creativecommons.org/ licenses/by-nc/4.0/

(c) Article author(s) (or their employer(s) unless otherwise stated in the text of the article) 2018. All rights reserved. No commercial use is permitted unless otherwise expressly granted.

\section{REFERENCES}

1 Hawe P, Potvin L. What is population health intervention research? Can J Public Health 2009;100:18-14

2 Shiell A, Hawe P, Gold L. Complex interventions or complex systems? Implications for health economic evaluation. BMJ 2008;336:1281-3.

3 Hawe P, Shiell A, Riley T. Theorising interventions as events in systems. Am J Community Psychol 2009;43:267-76.

4 Bisset S, Potvin L, Daniel M. The adaptive nature of implementation practice: case study of a school-based nutrition education intervention. Eval Program Plann 2013:39:10-18.

5 Tarquinio C, Kivits J, Minary L, et al. Evaluating complex interventions: perspectives and issues for health behaviour change interventions. Psychol Health 2015;30:35-51.

6 Cambon L, Minary L, Ridde V, et al. Transferability of interventions in health education: a review. BMC Public Health 2012;12:497.

7 Hawe P, Shiell A, Riley T. Complex interventions: how "out of control" can a randomised controlled trial be? BMJ 2004;328:1561-3.

8 Durlak JA, DuPre EP. Implementation matters: a review of research on the influence of implementation on program outcomes and the factors affecting implementation. $\mathrm{Am} J$ Community Psychol 2008;41:327-50.

9 Galbraith JS, Herbst JH, Whittier DK, et al. Taxonomy for strengthening the identification of core elements for evidence-based behavioral interventions for HIV/ AIDS prevention. Health Educ Res 2011;26:872-85.

10 Craig P, Dieppe P, Macintyre S, et al. Developing and evaluating complex interventions: the new medical research council guidance. BMJ 2008:337:a1655.

11 Clark AM. What are the components of complex interventions in healthcare? Theorizing approaches to parts, powers and the whole intervention. Soc Sci Med 2013;93:185-93

12 Shoveller J, Viehbeck S, Di Ruggiero E, et al. A critical examination of representations of context within research on population health interventions. Crit Public Health 2016;26:487-500. 
13 McCabe SE, West BT, Veliz P, et al. Social contexts of substance use among U.S. high school seniors: a multicohort national study. J Adolesc Health Off Publ Soc Adolesc Med 2014;55:842-4.

14 Poland B, Frohlich KL, Cargo M. Context as a fundamental dimension of health promotion program evaluation. In: Potvin L, McQueen DV, Hall M, de SL, AndersonLM, et al. eds. Health promotion evaluation practices in the Americas: Springer New York, 2008:299-317.
15 Pfadenhauer LM, Mozygemba K, Gerhardus A, et al. Context and implementation: A concept analysis towards conceptual maturity. Z Evid Fortbild Qual Gesundhwes 2015;109:103-14.

16 Rod MH, Ingholt L, Bang Sørensen B, et al. The spirit of the intervention: reflections on social effectiveness in public health intervention research. Crit Public Health 2014;24:296-307.

17 Bilodeau A, Potvin L. Unpacking complexity in public health interventions with the Actor-Network Theory. Health Promot Int 2016:daw062. 